\title{
Airframe Noise Simulations of a Full-Scale Aircraft
}

\author{
Jason Appelbaum, ${ }^{*}$ Benjamin Duda, ${ }^{\dagger}$ Ehab Fares ${ }^{\ddagger}$ \\ Exa GmbH, D-70563 Stuttgart, Germany \\ and \\ Mehdi R. Khorrami ${ }^{\S}$ \\ NASA Langley Research Center, Hampton, Virginia, 23681, USA
}

\begin{abstract}
Computational results for a full-scale simulation of a Gulfstream G-III aircraft are presented. In support of a NASA airframe noise flight test campaign, Exa Corporation's lattice Boltzmann PowerFLOW ${ }^{\circledR}$ solver was used to perform time-accurate simulations of the flow around a highly detailed, full-scale aircraft model. Free-air boundary conditions were used at a Mach number of 0.23 and a Reynolds number of $10.5 \times 10^{6}$ based on mean aerodynamic chord. This paper documents the simulation campaign for the baseline aircraft configuration at several flight conditions, including multiple flap deflections and main landing gear deployed or retracted. The high-fidelity, synthetic data were post-processed using a Ffowcs-Williams and Hawkings integral approach to estimate farfield acoustic behavior, with pressures on the model solid surface or a permeable surface enveloping the acoustic near field used as input. The numerical approach, simulation attributes, and the effects of grid resolution, gear deployment, and multiple flap deflections, are discussed as well.
\end{abstract}

\section{Nomenclature}

$\mathrm{C}_{\mathrm{P}} \quad=$ Pressure coefficient

$\mathrm{dp} / \mathrm{dt} \quad=$ Time derivative of pressure

$\operatorname{Re} \quad=$ Reynolds number based on mean aerodynamic chord

$\delta_{\mathrm{f}} \quad=$ Flap deflection angle

Acronyms

DNS $=$ Direct numerical simulation

ERA $=$ Environmentally Responsible Aviation

FDC $=$ Flight Demonstrations and Capabilities

FWH $=$ Ffowcs-Williams and Hawkings

ICAO = International Civil Aviation Organization

LBM = Lattice Boltzmann Method

MLG = Main landing gear

PSD $\quad=$ Power Spectral Density

RPK = Revenue passenger kilometers

SPL $\quad=$ Sound Pressure Level

VLES $\quad$ Very Large Eddy Simulation

\section{Introduction}

$\mathrm{N}$ oise produced by industrial environments, machinery and transportation poses an increasingly large risk to human and animal habitats worldwide. As the global population grows, and with it the reliance on mechanized systems of production and transportation, so does the potential for noise pollution. Global commercial airline passenger traffic is forecasted to grow by an average of more than $4 \%$ yearly over the next two decades, with the RPK (revenue passenger kilometers) more than doubling worldwide by 2035 [1]. Excess exposure to noise can have detrimental

\footnotetext{
*Application Engineer, Aerospace.

† Senior Application Engineer, Aerospace, Member AIAA.

¥ Senior Technical Director, Aerospace, Senior Member AIAA.

$\S$ Aerospace Engineer, Computational AeroSciences Branch, Associate Fellow AIAA.
} 
health effects on humans and worsen the quality of life in our communities, especially those near airports [2] or with particularly high population densities.

Increasingly, government regulatory action is taken to reduce this risk. One way in which governments and regulatory agencies are seeking to reduce environmental noise is by limiting noise produced by aircraft, especially during takeoff and landing where human impact is highest due to proximity to airports. The restrictions are now included in the standard certification process for aircraft and are expected to be made stricter in the future. For this reason, airframe producers are currently seeking new ways to reduce landing and takeoff noise without adversely affecting performance, and simulation tools such as the one discussed in this paper can greatly assist and guide this effort. The computational approach used in the present study is built upon the knowledge and experience gained from previous high-fidelity, aeroacoustic simulations of model- and full-scale complete Gulfstream aircraft that were shown to accurately predict the observed measured trends [3], [4], [5].

Development and advancement of system-level, simulation-based airframe noise prediction methodologies, first pursued under the NASA Environmentally Responsible Aviation (ERA) project, are being vigorously continued as part of the Flight Demonstrations and Capabilities (FDC) project of the NASA Aeronautics Research Mission Directorate. The main landing gears and the high-lift system are among the most dominant noise sources of conventional aircraft in landing configurations. The geometrical intricacies of these systems make them particularly difficult to simulate numerically because of the vastly different spatial and temporal scales involved and the highly complex spatial discretization required. Thus, a significant amount of effort was performed to obtain a high-fidelity CAD model of the G-III aircraft, involving laser-scanning of the complete aircraft surface and individual components to build a model accurate to within $\mathrm{O}(1 \mathrm{~mm})^{* * *}$ of the actual, flying, full-scale configuration with a $23.725 \mathrm{~m}$ wingspan.

This paper documents the simulation campaign for the baseline aircraft with various flap deflections and main landing gear deployed or retracted. The resulting high-fidelity, synthetic data were used to estimate farfield acoustic behavior. The numerical approach, simulation attributes, and the effects of grid resolution, gear deployment, and flap deflection are discussed as well.

\section{Baseline Model}

Except for the main landing gear, the baseline CAD model used for the simulations discussed in this paper was generated in support of the Adaptive Compliant Trailing Edge (ACTE) flap, steady aerodynamic load calculation [6], [7]. The model was obtained from laser scans of the surface of a full-scale G-III aircraft. Since capturing the local geometric details is of paramount importance for airframe noise simulations, an extensive effort was made to benchmark the laser-scanned data against the actual aircraft features and dimensions, and to add any omitted elements. The latter included reconstruction of the flap bracket assemblies, tracks, vane guides, shallow cavities at the inboard and outboard side-edges, and a host of other features. The surface definition for the hush kit, which is a recent addition to the G-III engines, was developed from information and engineering drawings provided by the manufacturer and from physical inspection of the aircraft.

For the current study, a highly defined geometry of the main landing gear, including its cavity, was painstakingly developed from carefully conducted laser scans of the component while the aircraft was placed on jacks to have the gear in the fully extended landing configuration. Significant effort was spent ensuring that the finest details of the main gear exterior surfaces (i.e., outer mold lines) were captured accurately. However, a major deficiency of this type of geometry reconstruction is the lack of proper definition for some of the internal cavities that may be present on some of the gear subelements, which could potentially contribute to the tonal component of the gear noise signature. Fortunately, for the G-III main landing gear, only the opening in the front post, which runs the entire length of the component, could not be mapped out with the laser scanning process. The internal opening in this post was reconstructed via direct measurements of a few critical locations and features of this gear component. As a result, the frequency of the predicted tone generated by the post cavity was noticeably different than the frequency measured during the flight test - a direct result of the mismatch between modeled and "as flown" volume of the opening in the front post of the main gear [8].

As can be seen in Figure 1, almost all major components of the aircraft are included in the simulation model, i.e., fuselage, wing with deflected flaps including brackets, tail, nacelle with hush-kit, and main landing gear. The nose landing gear was omitted to isolate the effects of main landing gear and flap. Since this study focuses on airframe noise, a flow-through nacelle was used.

All elements of the CAD model were surface-meshed using PowerDELTA® and integrated into the computational model. Great care was taken to ensure the accuracy of the surface mesh tessellation. The maximum allowed spatial deviation between the CAD and the discretized surface mesh was $0.01 \mathrm{~mm}$ for all components in areas of high

\footnotetext{
** This accuracy was maintained only for the components critical to airframe noise.
} 
curvature, and $0.05 \mathrm{~mm}$ for larger scale areas such as the fuselage. This represents $2.4 \times 10^{-4} \%$ and $1.2 \times 10^{-3} \%$ of the Mean Aerodynamic Chord, respectively. Flap geometry for the three flap deployment settings used throughout the simulation campaigns are depicted in Figure 2.
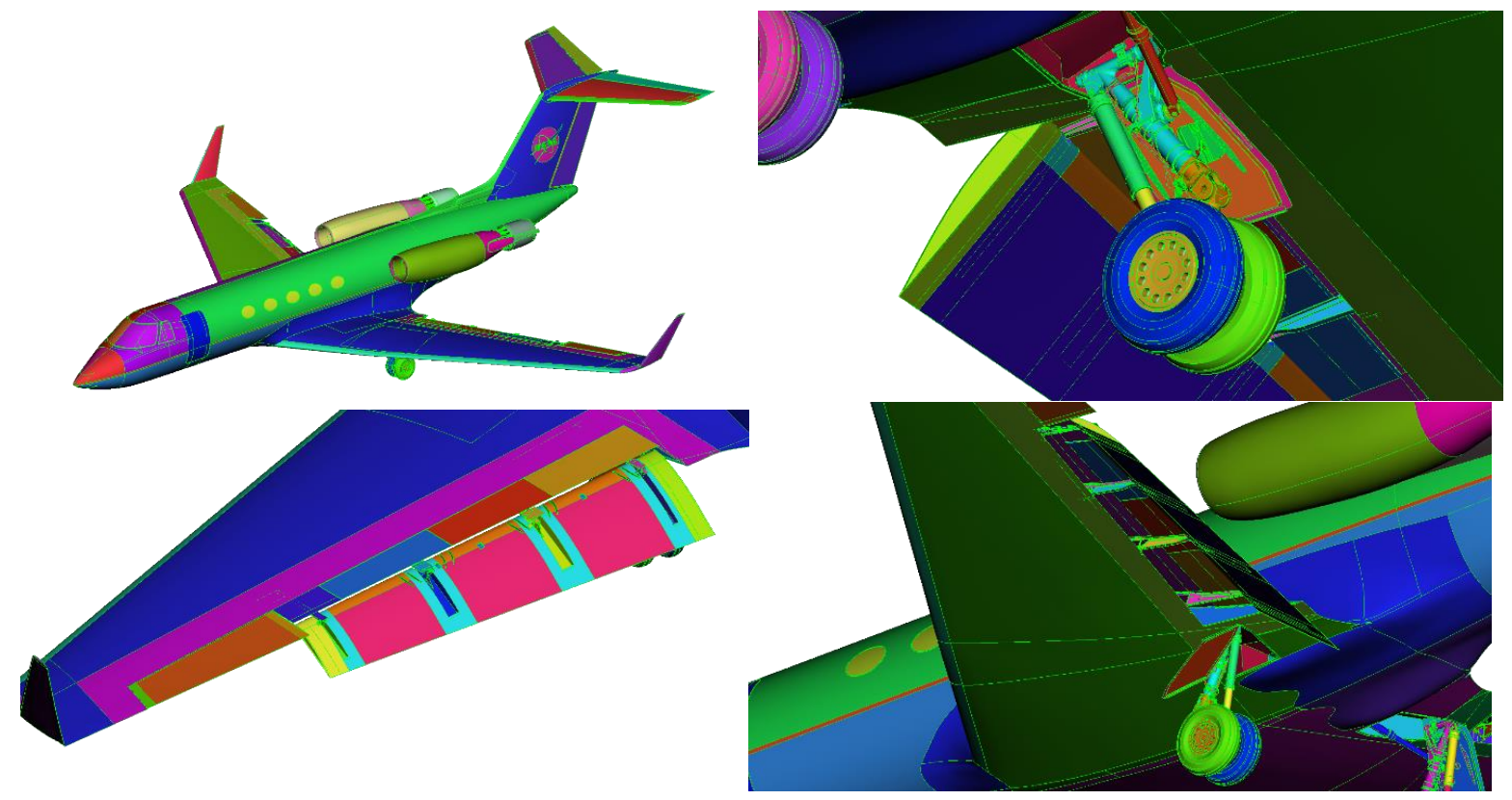

Figure 1 Gulfstream G-III aircraft CAD model.

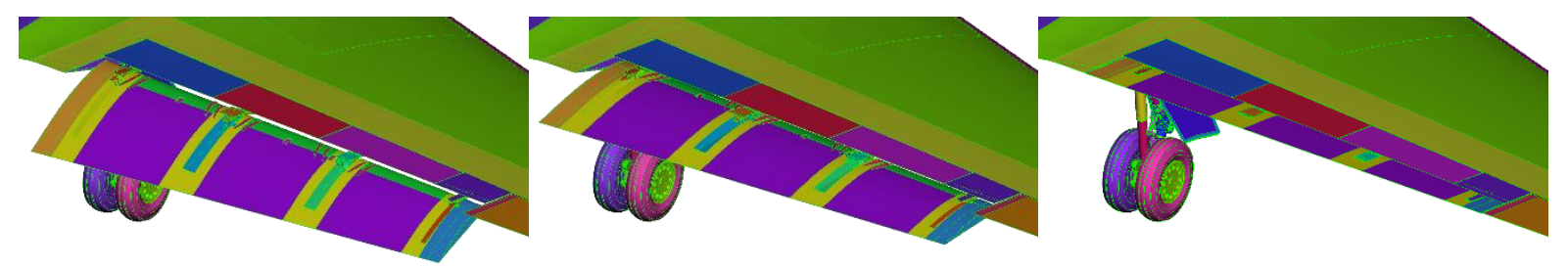

Figure 2 G-III Flap deployments (from left to right): $\delta_{\mathrm{f}}=3^{\circ}, \mathbf{2 0}^{\circ}$ and $0^{\circ}$ (stowed).

\section{Computational Approach}

The numerical simulations were performed using Exa Corporation's PowerFLOW ${ }^{\circledR}$ solver, which is based on the three-dimensional, 19-state (D3Q19) lattice Boltzmann method (LBM) [9], [10], [11], [12]. LBM has been extensively validated for a wide variety of applications ranging from academic direct numerical simulation (DNS) cases to industrial flow problems in the fields of aerodynamics [13] and aeroacoustics [3], [14], [15]. At a macroscopic level, LBM uses a simpler and more general physics formulation than methods based on the Navier-Stokes equations [9]. The LBM equations recover the macroscopic hydrodynamics of the Navier-Stokes equations through the ChapmanEnskog expansion [16], [17].

The local formulation of the LBM equations allows a highly efficient implementation for distributed computations on thousands of processors. The low dissipation and dispersion properties of the numerical scheme typically produce aerodynamic and aeroacoustic results that are comparable to those obtained with classical CFD solvers that use higherorder large eddy simulation (LES).

\section{A. Turbulence Modeling}

The lattice Boltzmann flow simulation is equivalent to a DNS of the flow. For high Reynolds number (Re) flows, such as those addressed in this work, the lattice Boltzmann Very Large Eddy Simulation (LB-VLES) approach is used to reduce computational resource requirements [11], [18]. This means that turbulence is modeled in areas of attached flow such as boundary layers but resolved in wakes or regions of detached flows. 


\section{B. Wall Treatment}

The standard lattice Boltzmann bounce-back boundary condition for no-slip or the specular reflection for free-slip condition are generalized through a volumetric formulation [9], [10] near the wall for arbitrarily oriented surface elements (surfels) within the Cartesian volume elements (voxels). This formulation of the boundary condition on a curved surface cutting the Cartesian grid is automatically mass, momentum, and energy conservative while maintaining the general spatial second-order accuracy of the underlying LBM numerical scheme. To reduce the resolution requirements near the wall for high Reynolds number flows, a hybrid wall function is used to model the region of the boundary layer closest to the solid surfaces [13], [19].

\section{Complex Geometry Handling and Meshing}

The lattice Boltzmann approach is solved on Cartesian meshes. Variable resolution regions (VR) can be defined to allow for local mesh refinement of the grid size by successive factors of two. Based on the facetized geometry and the local volume resolution, the model surface is discretized by planar surfels. This process allows the automatic generation of computational grids for any arbitrarily complex geometrical shapes. To illustrate the numerical grid, a slice cutting through the gear and the inboard tip of the deflected flap is shown in Figure 3. Depending on geometrical and flow structure complexity, areas of high resolution have been defined to properly resolve the flow field. Only every other mesh line is shown for clarity.

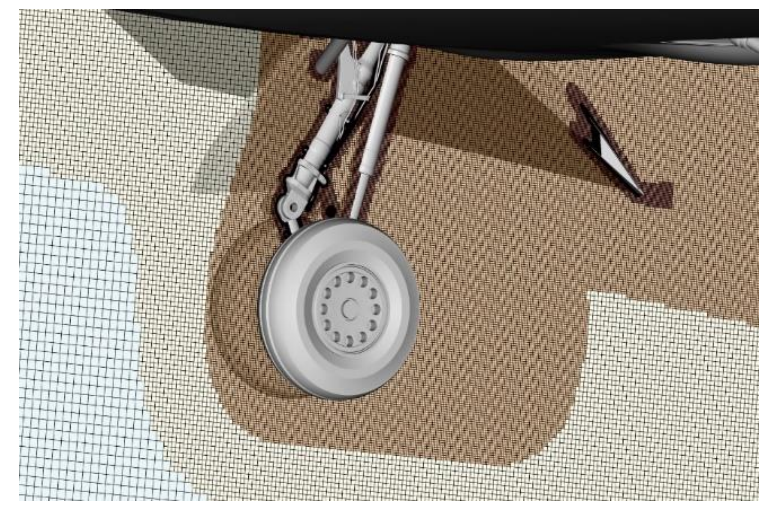

Figure 3 Volume mesh in the vicinity of main gear and flap showing different resolution levels.

\section{Simulation Setup}

All simulations of the baseline campaign were performed at full scale and with free-air boundary conditions. A Mach number of 0.23 and a Re of $10.5 \times 10^{6}$ were used for the computations. The latter represents a value that is close to $60 \%$ of the flight Re based on the mean aerodynamic chord of $13.78 \mathrm{ft}(4.2 \mathrm{~m})$ and an aircraft speed of 150 knots. This chosen Re is sufficiently high to produce farfield noise levels that are nearly equivalent, at a lower computational cost, to those obtained at full flight Re [20]. The simulation domain was initialized with free flow conditions except in the immediate near field region around the aircraft, which was initialized with zero flow velocity and free stream pressure. For the first several cases run, a symmetry plane normal to the lateral direction was used to reduce computational cost. The effect of using the symmetry plane is discussed in section IV C.

The initial $\sim 0.60 \mathrm{~s}$ of simulated physical time represents the transient to settled flow conditions and is not used for statistical sampling. After this initial time segment, various data are recorded from the simulation, most importantly the high-frequency sampled pressure field on the surface of the aircraft and the density, velocity and pressure field on a permeable surface outside of the acoustic near field. These datasets are written out for $\sim 1.30 \mathrm{~s}$ of simulated physical time. These two main measurements are eventually used in conjunction with Exa's far field noise solver within PowerACOUSTICS ${ }^{\circledR}$ to obtain pressure signals at defined locations.

\section{E. Measurement Entities}

The main acoustic measurements that define the dataset that is ultimately written out for post-processing are the solid surface fluctuating pressure field, or "solid FWH" measurement, and a sampled permeable data surface enveloping the acoustic near field upon which pressure, velocity and density are recorded, or the "permeable FWH" measurement, as shown in Figure 4. The definition of this surface is based on the requirement that hydrodynamic fluctuations crossing its boundaries should be minimal. To achieve this, an offset surface was generated that 
encompasses all areas of high root-mean-square (RMS) values of velocity. Both solid and permeable measurements were sampled with a high frequency, corresponding to the physical time step on the local mesh, for the entirety of the acquisition segment having a duration of $\sim 1.30 \mathrm{~s}$.

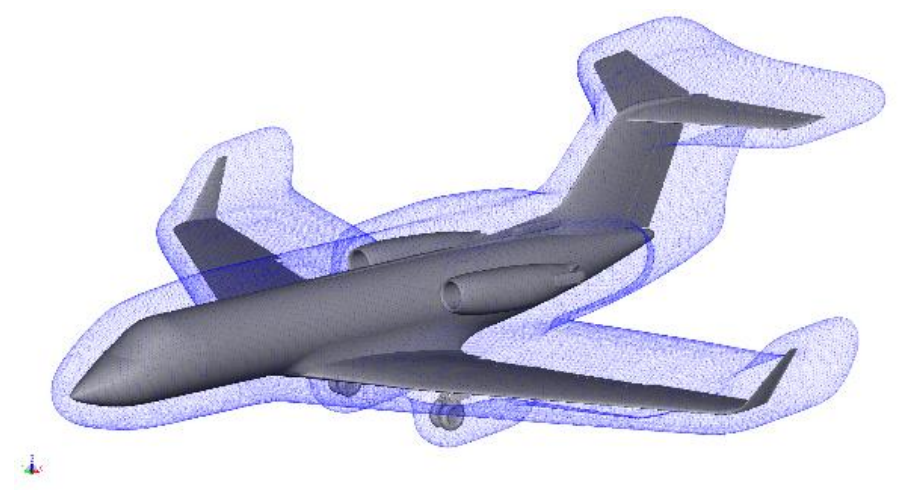

Figure 4 "Coarse wrap" used for the permeable FWH measurement.

\section{F. Farfield Noise Solver}

An acoustic analogy approach based on the Ffowcs-Williams and Hawkings (FWH) formulation [21] was used to propagate the computed nearfield fluctuations to the far field via the efficient and well-validated formulation developed by Farassat [22], also known as formulation 1A. The formulation is extended to account for uniform mean flow convection effects to simulate the noise generated and measured in an ideal infinite wind tunnel [23].

\section{G. EPNL}

The Effective Perceived Noise Level (EPNL) was calculated using a proprietary post-processing tool developed by Exa Corporation. The primary reference for the definition and calculation of EPNL is a publication of the ICAO [24]. The input to the tool is a semicircular arc of FWH signals. Once a trajectory is determined, it is discretized into flight segments of a given duration, in this case $500 \mathrm{~ms}$. For every flight segment, the emission time and position of the aircraft is determined, and a ray between the observer and aircraft is traced. The ray's intersection with the semicircular microphone array is determined and a noise spectrum for the observer is formulated by interpolating the appropriate microphones' narrow-band spectra. The spectrum at the observation point is corrected for distance, atmospheric absorption, Doppler shifting and ground reflection for each flight segment. One-third octave sound pressure levels (SPL) are then computed and Perceived Noise Level (PNL) is calculated using the procedure described by the ICAO. Finally, tonal weighting and band sharing adjustments are made and EPNL is computed.

\section{Numerical Results}

\section{A. Grid Resolution Study}

To assess the dependence of the numerical solution on the underlying volume grid, results obtained for different resolution levels will be presented in this section. The global resolution, i.e., the edge length of a voxel, is scaled consecutively by a factor of $1 / 1.5$. The resulting three simulations are termed "coarse", "medium" and "fine".

The full-scale baseline geometry, i.e., both main gear and flap deployed, was run at an angle of attack of $6^{\circ}$, a Mach number of 0.23 and a $\operatorname{Re}$ of $10.5 \times 10^{6}$. The resulting mesh sizes and required timesteps are summarized in Table 1.

Table 1 Simulation metrics for baseline configuration resolution study.

\begin{tabular}{lcclcc} 
Resolution & Voxels $\left[10^{6}\right]$ & Surfels $\left[10^{6}\right]$ & Min Edge $\left[10^{-4} \mathrm{~m}\right]$ & Timesteps & CPUh $\left[10^{6}\right]$ \\
\hline Coarse & 1711 & 41 & 7.2 & 2293760 & 0.2 \\
Medium & 5436 & 72 & 4.8 & 3440640 & 1.2 \\
Fine & 17436 & 126 & 3.2 & 5177344 & $\approx 8.5$ \\
\hline
\end{tabular}

A qualitative view of the flow field around the wing and gear are shown in Figure 5, where an isosurface of averaged total pressure is included in yellow. Clearly visible are the flap side edge vortices as well as the two large flow separation zones produced by the flap middle brackets. An additional view of the gear shows the wake topology. Differences in the overall flow topology due to resolution are minor for all three resolutions. A qualitative comparison of pressure distribution at three different span sections is shown in Figure 6 for coarse, medium and fine resolutions. 
Again, differences between these three results are minimal which, at least for the mean pressure field, indicate good grid convergence.

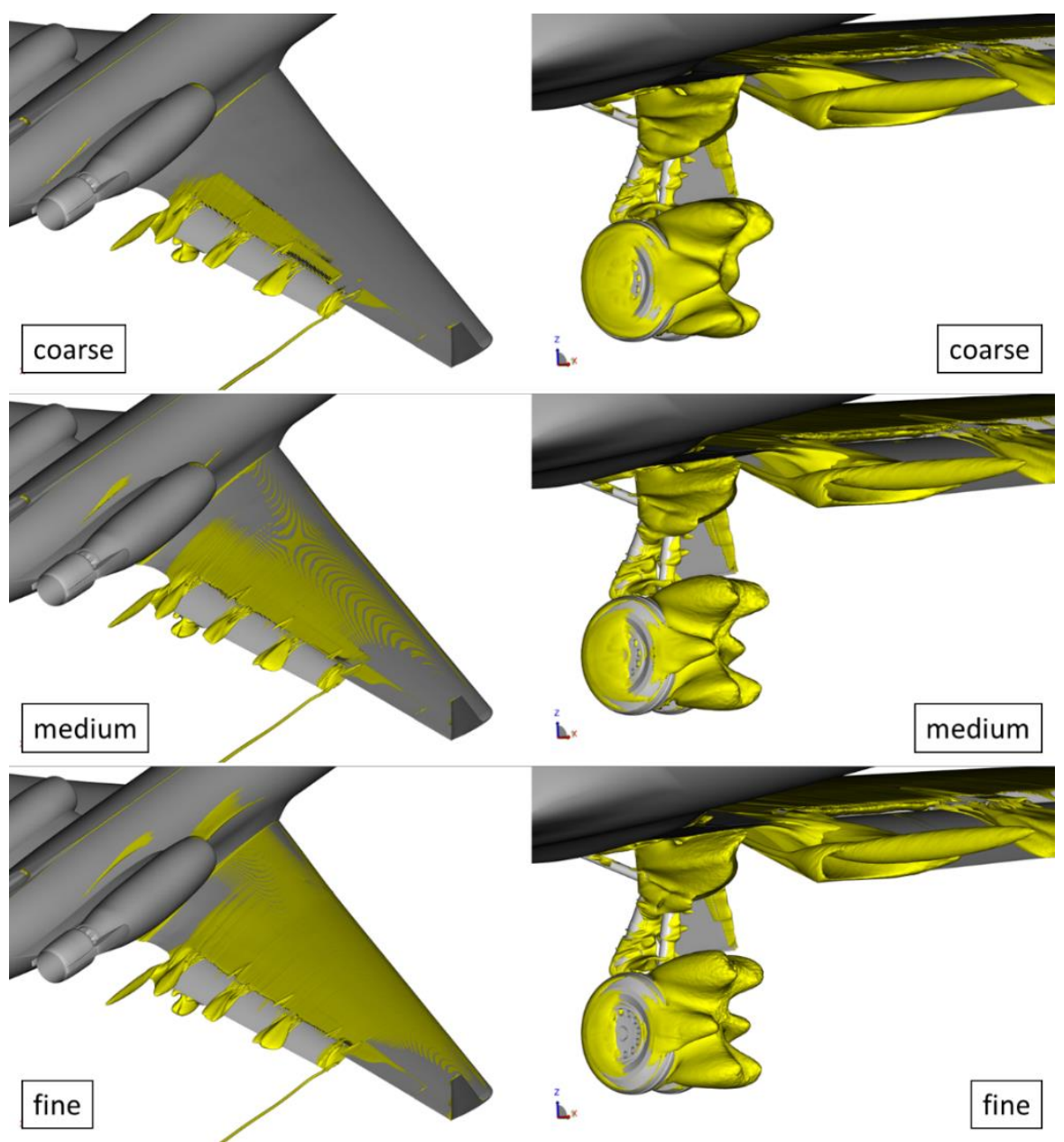

Figure 5 Isosurfaces of $C_{p, t}=0$ showing flap side edge vortices and flow separation zones on flap (left) and gear (right) for coarse (top), medium (middle) and fine (bottom) resolutions for flap deflection angle $\delta_{\mathrm{f}}=\mathbf{2 0}^{\circ}$.
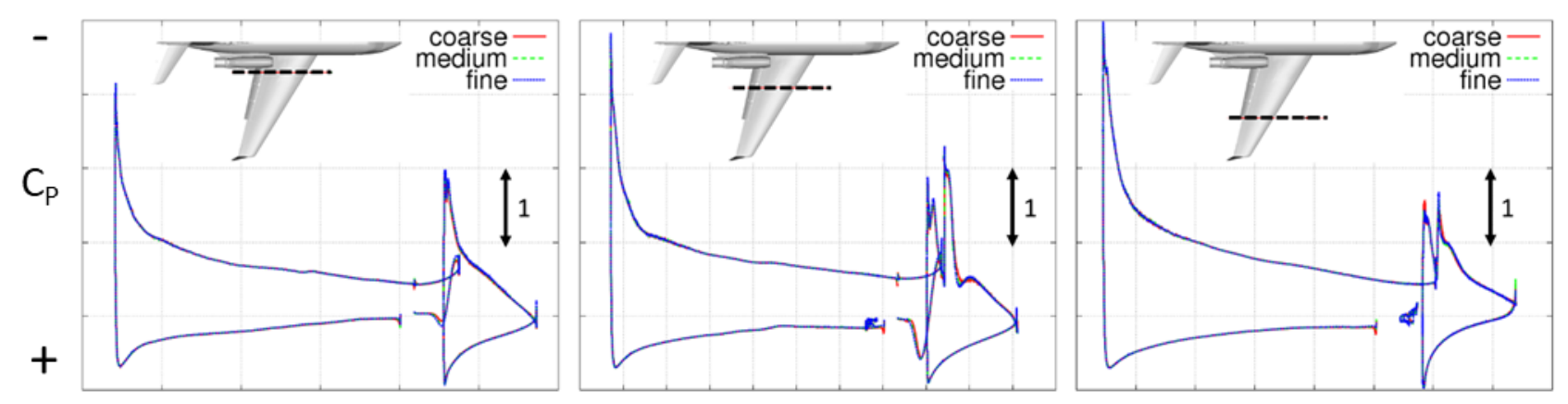

Figure 6 Pressure distribution at three different spanwise locations for coarse (solid red), medium (dashed green) and fine (dotted blue) simulation for flap deflection angle $\delta_{\mathrm{f}}=\mathbf{2 0}$.

Higher-order time statistics such as root-mean-square (RMS) values of pressure are recorded as well. They give an indication of the unsteadiness of the flow and can be connected to acoustic noise sources. Figure 7 shows a closeup view of the flap where areas of high pressure fluctuations can be observed at the flap side edges and flap brackets for all three resolution levels. Differences in shape and intensity for this quantity are visible, which are due to increased 
resolution of turbulent fluctuations due to the finer meshes. Interestingly, there are also regions of increased fluctuation levels close to the trailing edge of the main wing, which are caused by the actual spoiler gaps in the geometry.

Another insight into the time-dependent flow field is given by a slice in the vicinity of the main gear. Instantaneous values of velocity magnitude, shown in Figure 8, indicate a highly turbulent and unsteady flow in the wake of the gear. Since the plane is also cutting through the inboard edge of the flap, the unsteadiness shown there is connected to the shear-layer roll-up and turbulent flow structures being drawn into the flap side edge vortex. As can be seen, the interaction of the main gear and the flap side edge is strong, which necessitates a simulation on a system level to ascertain the complex component interaction (landing gear installation) effects. As expected, more and finer structures are resolved as the grid resolution increases.

The time derivative of pressure is calculated and shown in Figure 9 on a plane cutting through the flap, as a qualitative depiction of pressure wave generation and propagation. Clearly visible are the prominent noise sources, i.e., flap side edges, flap brackets, and main gear. As expected, a better spatial resolution of the flow also leads to a better resolved acoustic field.

Finally, attention is turned toward farfield acoustics. In this case, the FWH approach based on the solid formulation has been used to obtain pressure data at a virtual microphone located directly below the aircraft at a distance of 120 $\mathrm{m}$. Since sampling frequency directly depends on mesh resolution, pressure has been sampled at $26.6 \mathrm{kHz}$ for the coarse simulation, at $40 \mathrm{kHz}$ for the medium simulation, and at $60 \mathrm{kHz}$ for the fine simulation. The power spectral density and the corresponding one-third octave SPL have then been calculated and are plotted in Figure 10. As expected, increased spatial and temporal resolution leads to more high frequency content, whereas the low frequency range is largely unaffected. The peak at around $550 \mathrm{~Hz}$ is attributed to the resonant response of the hollow front post of the MLG to the unsteady grazing flow at the cavity opening. Interestingly, this peak diminishes in the fine simulation but is still present at other microphone positions (directivity angles) that are not shown here. The most likely cause of the reduction in peak amplitude are small differences in the mean flow topology and unsteady flow at the post's cavity opening that result from additional resolution of turbulent structures in the fine grid. Finer spatial and temporal resolutions allow for more degrees of freedom in the unsteady flow, allowing previously coherent flow structures responsible for exciting the resonant tone such as shed vortices to destabilize.
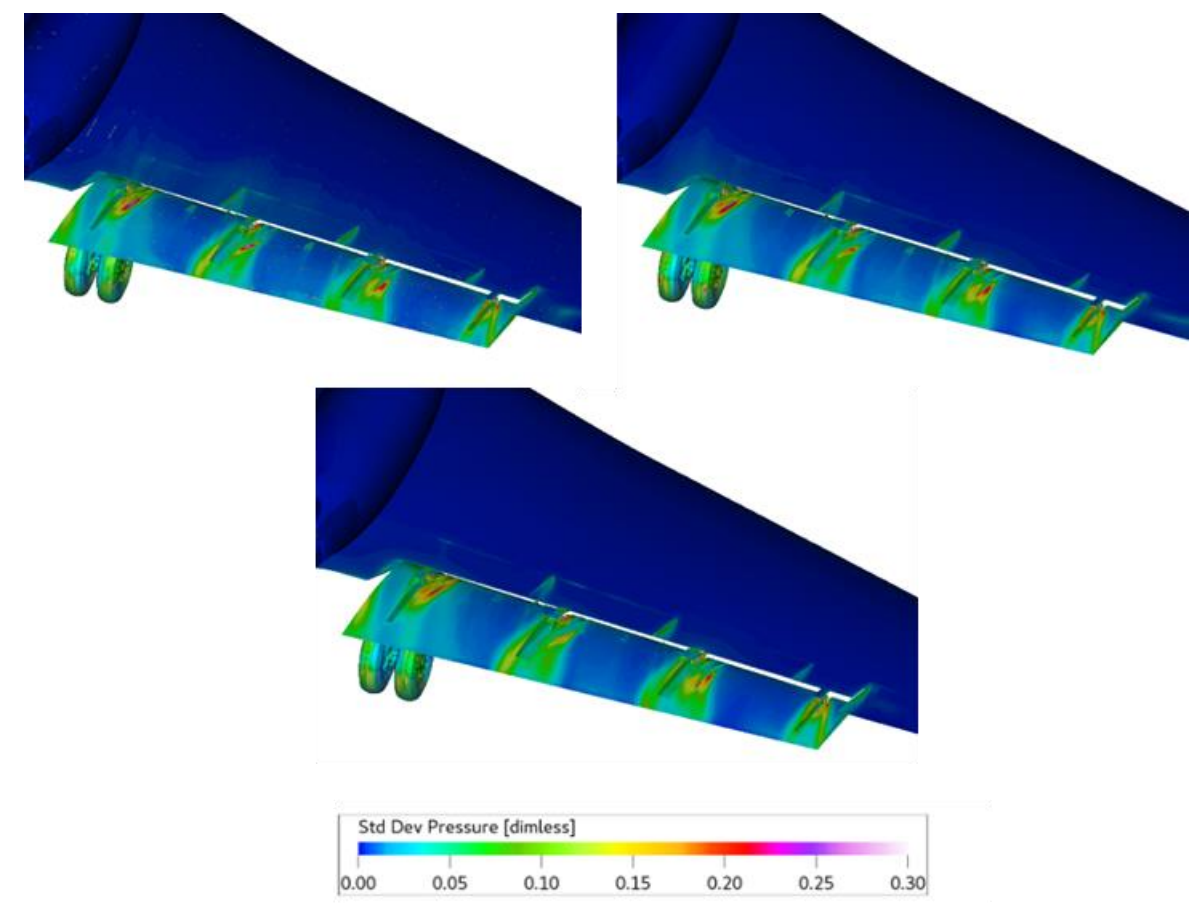

Figure 7 Pressure RMS distribution on wing and flap for coarse (top left), medium (top right) and fine (bottom) simulation for flap deflection angle $\delta_{\mathrm{f}}=\mathbf{2 0 ^ { \circ }}$. 


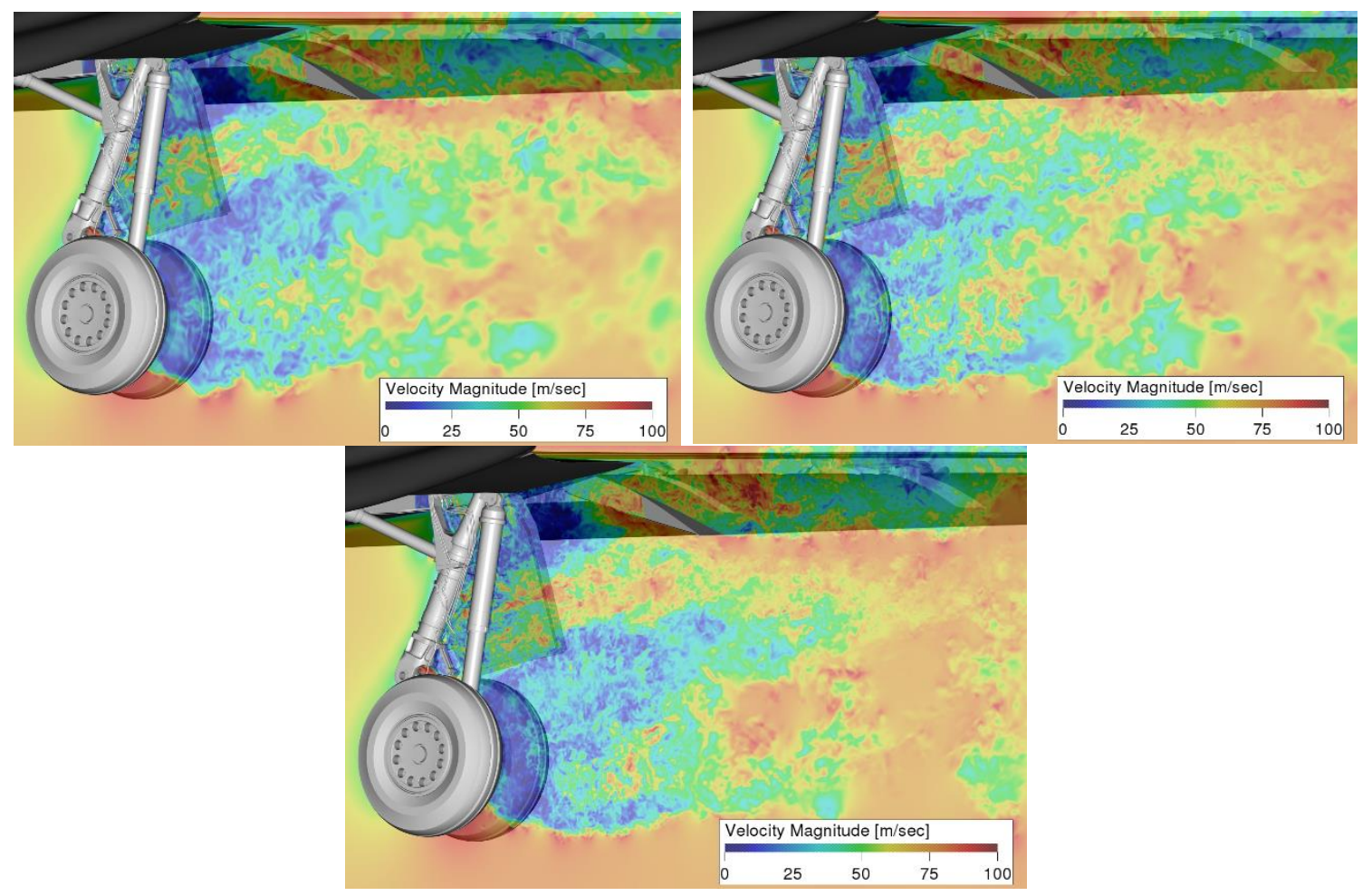

Figure 8 Instantaneous velocity on a plane cutting through gear and flap side edge for coarse (top left), medium (top right) and fine (bottom) run for flap deflection angle $\delta_{\mathrm{f}}=\mathbf{2 0}$.
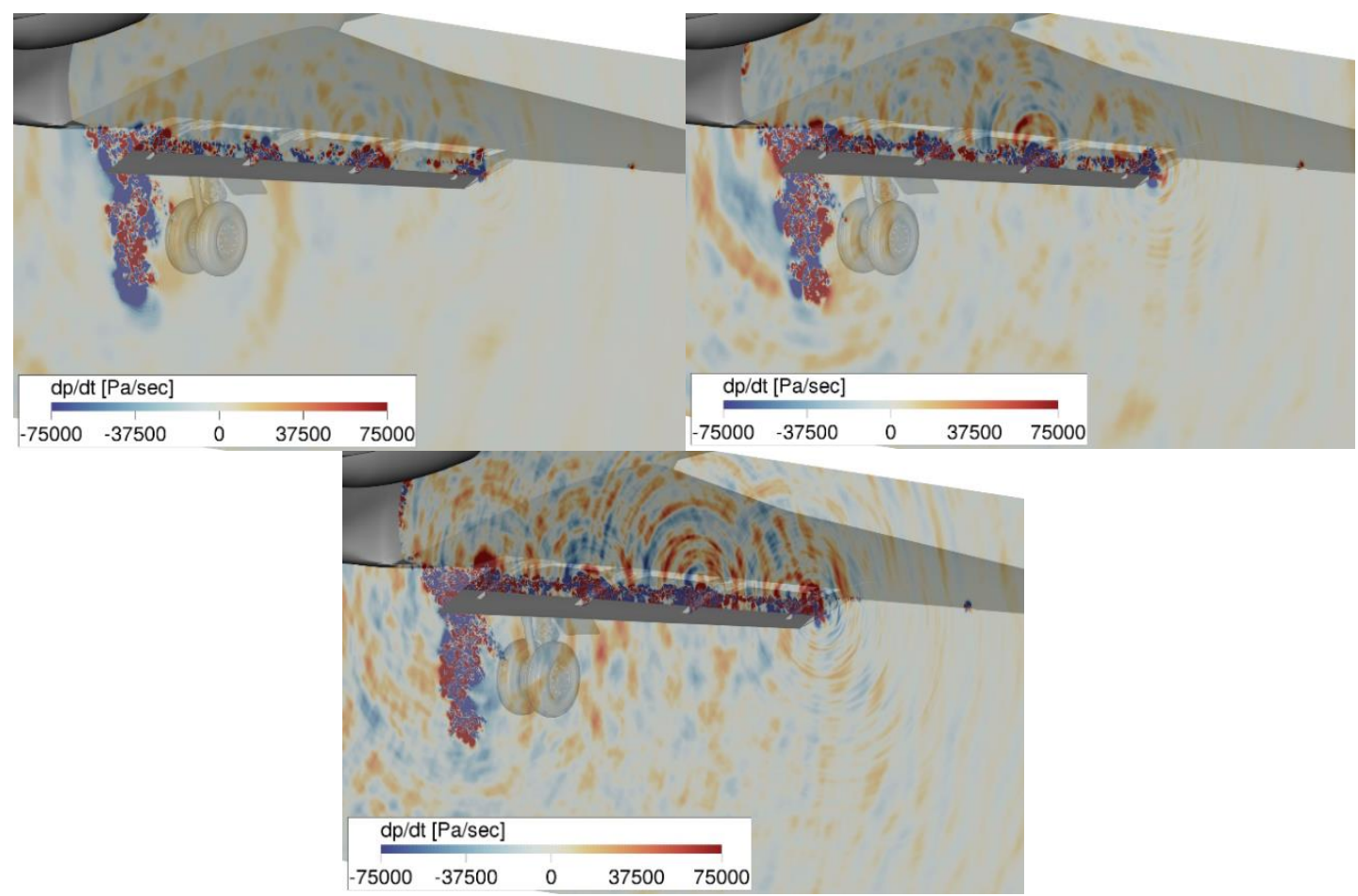

Figure 9 Time derivative of pressure on plane cutting through flap for coarse (top left), medium (top right) and fine (bottom) simulation for flap deflection angle $\delta_{\mathrm{f}}=\mathbf{2 0 ^ { \circ }}$. 

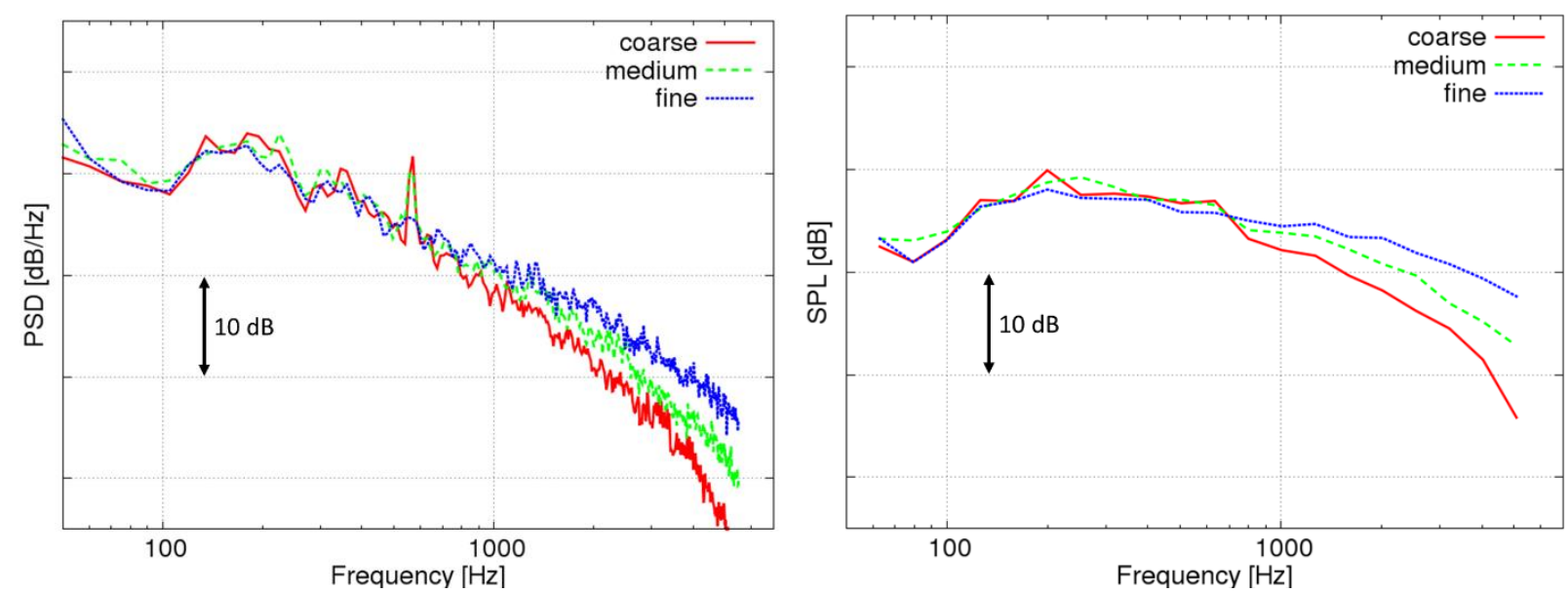

Figure 10 Power spectral density (left) and 1/3rd octave SPL (right) for coarse (solid red), medium (dashed green) and fine (dotted blue) simulation for flap deflection angle $\delta_{\mathrm{f}}=\mathbf{2 0}$.

\section{B. Direct Probe in Comparison with Solid and Permeable FWH Approach}

To corroborate the accuracy of the farfield signals acquired through the FWH solver, a test was constructed to compare to a direct probe, where the unsteady pressure field underneath the aircraft is captured directly within the simulation without any use of the farfield propagation tool, as shown in Figure 11. To accomplish this task, a region of high resolution mesh was extended up to $12 \mathrm{~m}$ (highlighted with green lines) to several farfield points. The same resolution used for the permeable FWH measurement surface was applied to the extended region (see Figure 12).

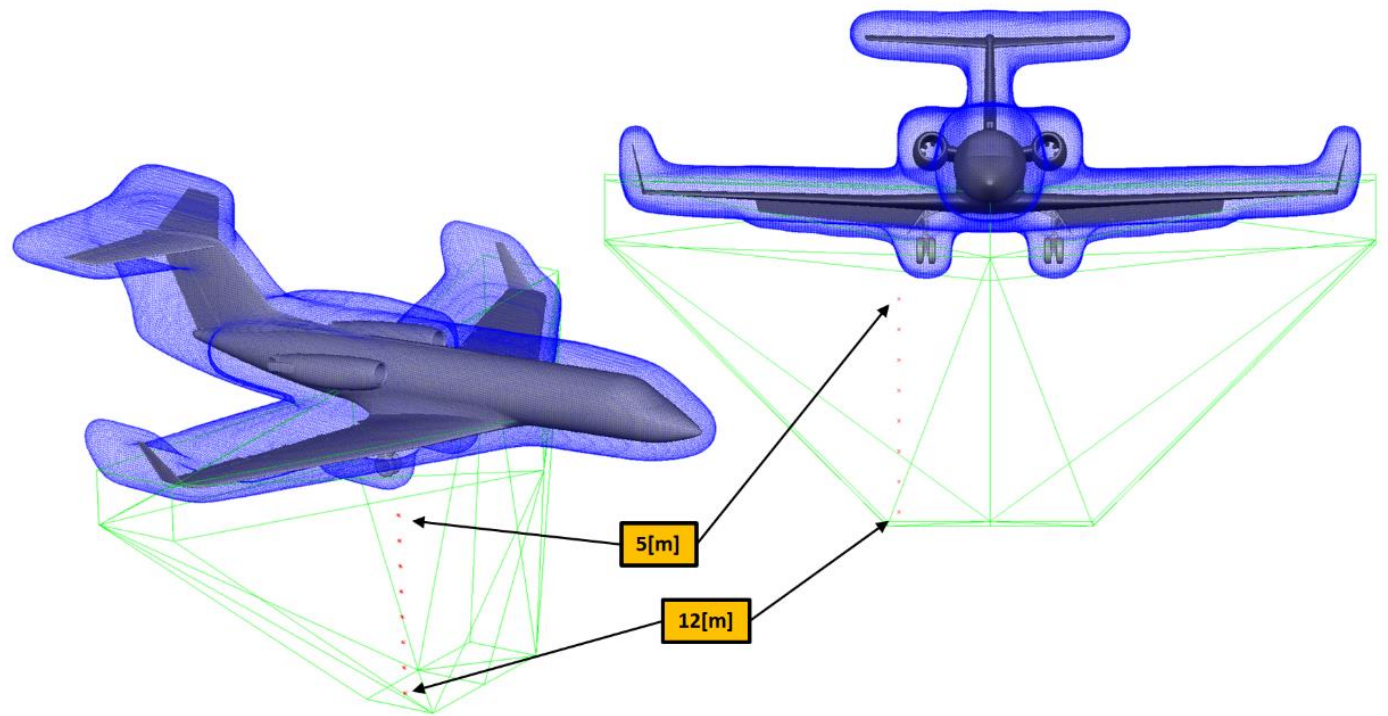

Figure 11 High resolution direct probe regions and FWH surface, aircraft in landing configuration with flap deflection angle $\delta_{\mathrm{f}}=39^{\circ}$. 


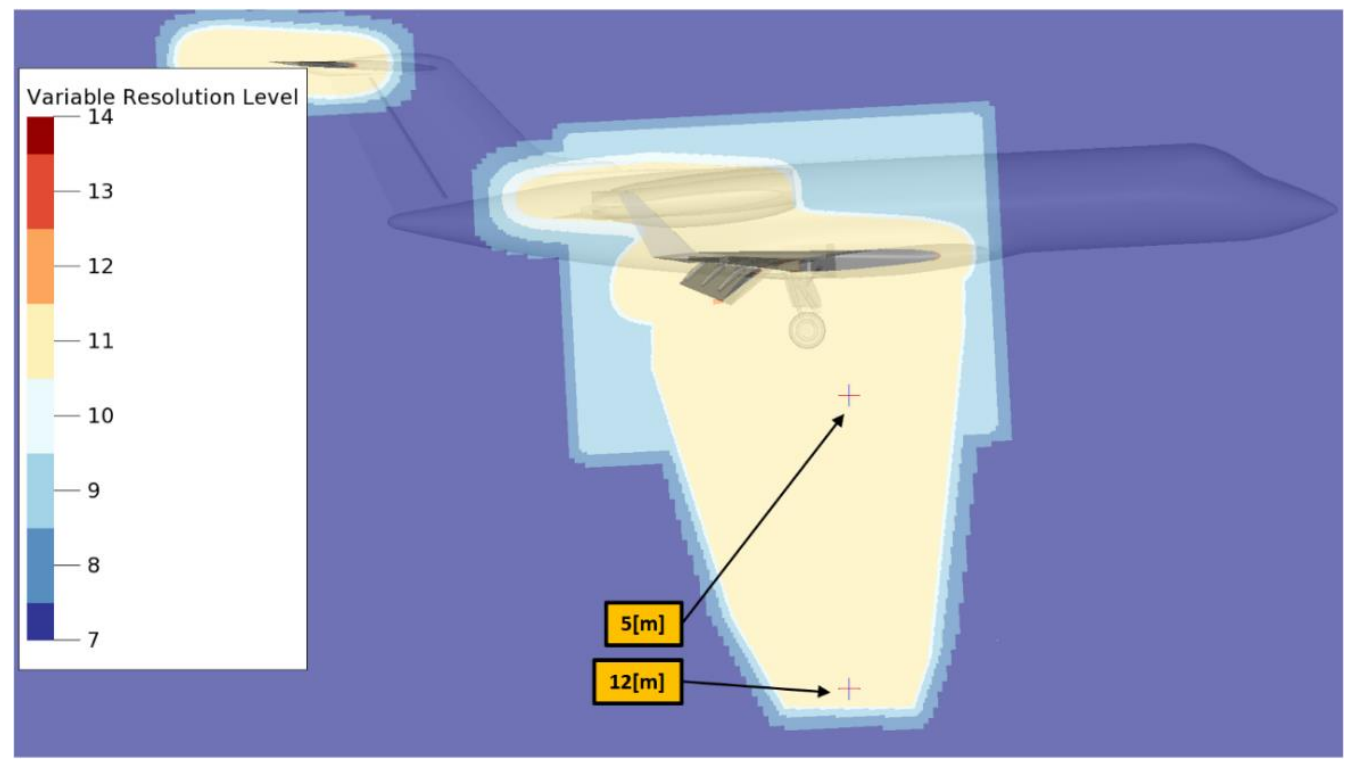

Figure 12 Variable resolution levels at $\mathbf{y}=\mathbf{3} \mathrm{m}$ slice.

Figure 13 depicts comparisons for a probe located $5 \mathrm{~m}$ and $12 \mathrm{~m}$ under the aircraft, as shown in Figures $\mathbf{1 1}$ and 12. The comparisons at $5 \mathrm{~m}$ indicate a good match between the direct probe signal and the propagated signal based on the permeable surface up to $\sim 2500 \mathrm{~Hz}$, whereas the propagation based on the solid surface is at higher sound pressure levels. At the larger distance of $12 \mathrm{~m}$, good agreement is shown for all spectra up to $\sim 800 \mathrm{~Hz}$, above which the direct probe shows a lower sound pressure level related to the grid cut-off of the directly propagated acoustic wave. The solid and permeable FWH surfaces show good agreement up to roughly $1500 \mathrm{~Hz}$; for higher frequencies the permeable FWH surface shows lower SPL, again due to diffusion effects from mesh resolution. Based on these results and other previous studies [25] highlighting the importance of volumetric effects, the permeable surface was used for farfield noise propagation.
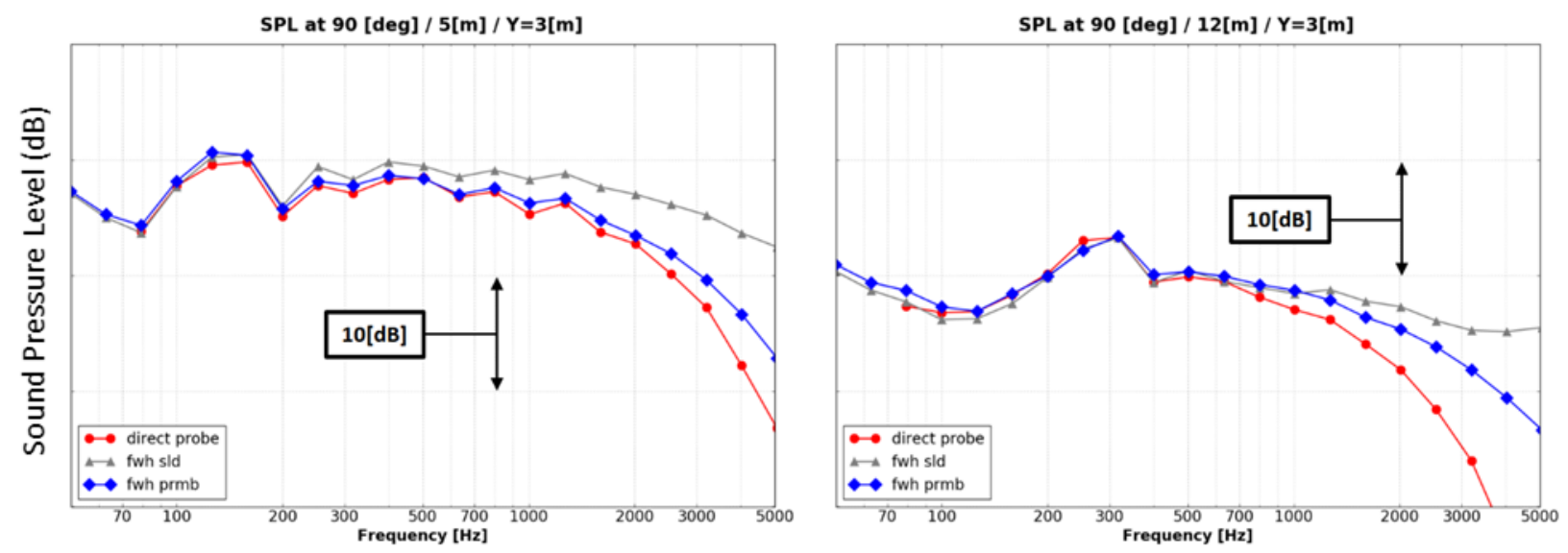

Figure 13 Comparison of 1/3rd octave SPL for FWH surfaces and direct probe at distances of $5 \mathrm{~m}$ (left) and $12 \mathrm{~m}$ (right) for medium simulation.

\section{Effect of Symmetry Plane}

At the beginning of the simulation campaign, most of the runs were conducted with a symmetry boundary condition for expediency. The symmetry plane, however, does not allow crossing of turbulent fluctuations nor acoustic waves. The fluctuations will be damped, whereas the waves will be reflected entirely. This can be problematic if the gears are located in close proximity of each other or if the gear cavity is connected. The latter is the case for the aircraft 
considered here, where symmetrical and antisymmetrical cavity modes are present and are important for low frequency noise characterization.

Two simulations, with and without a symmetry plane, were conducted using a coarse resolution. The power spectral densities for a farfield pressure probe located at a forward angle of $45^{\circ}$ and at a distance of $120 \mathrm{~m}$ away from the aircraft are shown in Figure 14. To compare half- and full-span simulations, only the right half of the full-span geometry has been accounted for in the farfield propagation computation and the solid aircraft surfaces were used in the FWH approach. As can be seen, the two results are similar with the exception of a tone appearing at about $170 \mathrm{~Hz}$ for the full-span simulation. This tone, however, is not present when the entire full-span geometry is used in the FWH computation. It is worth noticing that, for mid to high frequencies, a reduction of roughly $3 \mathrm{~dB}$ is visible when comparing the entire full-span contribution with only the right half contribution of the same simulation. This indicates that airframe noise generated on each side of the aircraft represents two uncorrelated noise sources.

Since the low frequency tones are very likely connected to the MLG cavity, a bandpass filter was applied to the surface pressure fluctuations. The results for each of the three frequency bands representing a tone are shown in Figure 15. The shapes of the first and third cavity modes are symmetrical and appear for both the half- and the full-span simulation, which explains the first and third peak in the spectra shown in Figure 14. However, the second cavity mode is antisymmetrical and thus, only present in the full-span simulation. Since the emitted pressure waves at each of the gear openings are phase-shifted by $180^{\circ}$, their contribution cancels out in the far field. If only half of the geometry is considered, no cancellation takes place and the second tone in the spectrum appears. Note that the strong tones produced by the cavity exist for the simulated geometry only. In reality, the cavity hosts a large number of auxiliary components such as hydraulic pipes and other devices, which most likely destroy or significantly diminish any cavity resonance. For this reason, a small sponge zone of artificially high viscosity was introduced into the cavity ceiling for future simulations because it reduces the tones in the simulation without having to replicate the entire cavity geometry.

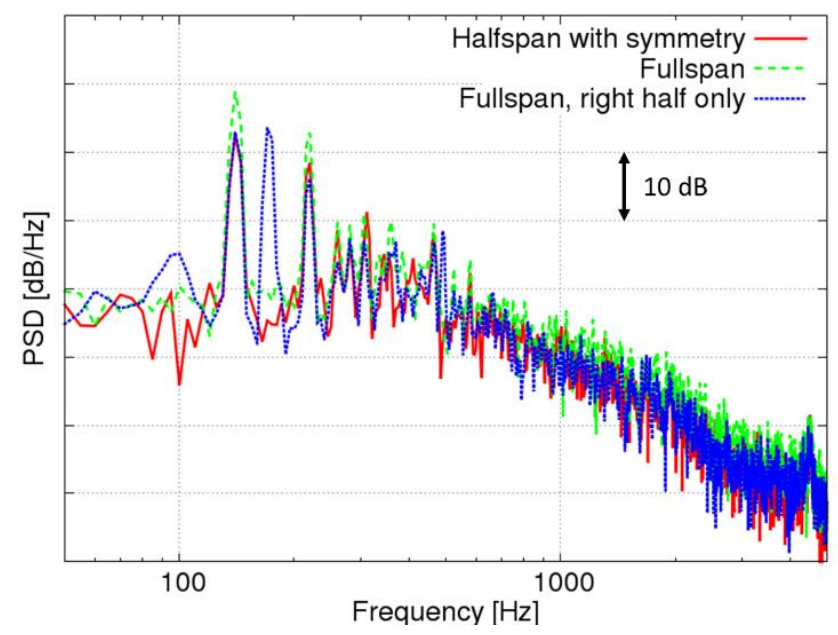

Figure 14 Power spectral density of farfield probe for half-span simulation using symmetry plane (solid red), fullspan simulation (dashed green) and fullspan simulation with contribution of right half only (dotted blue). 

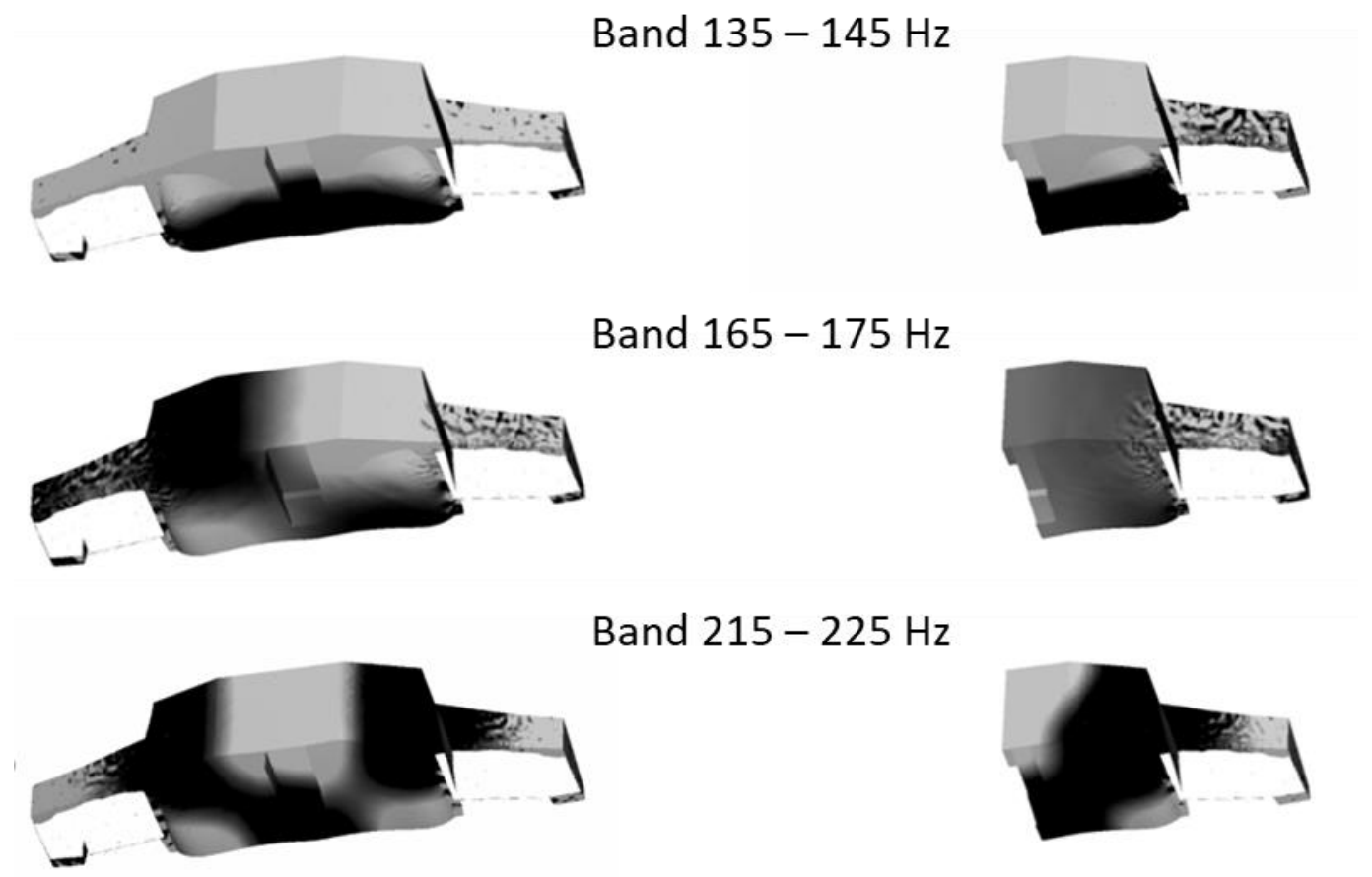

Figure 15 Band pass filtered surface pressure inside the MLG cavity for full-span (left) and half-span (right) simulation for three different bands: $135-145 \mathrm{~Hz}$ (top), $165-175 \mathrm{~Hz}$ (middle) and 215-225 Hz (bottom).

\section{Impact of Flap and Gear Deflection on Overall Noise}

Since gear and flap noise interact and the latter is dependent on the deflection angle, multiple simulations were carried out to assess the full ramifications of the complex component interaction effects. All three flap deflection angles, i.e., $\delta_{\mathrm{f}}=0^{\circ}$ (stowed), $20^{\circ}$ and $39^{\circ}$, have been simulated with retracted and deployed MLG on a medium resolution grid. Note, however, that the stowed flap configurations were simulated with an angle of attack reduced by $1.25^{\circ}$.

The impact of MLG deployment and flap deflection on aerodynamics was investigated independently. Figure 16 shows the pressure distribution at three different spanwise locations for a simulation with and without MLG and a flap deflection angle $\delta_{\mathrm{f}}=20^{\circ}$. The impact of the MLG on wing and flap aerodynamics is very small and only limited to the vicinity of the MLG. Figure 17 shows the impact of Fowler flap deflection. As expected, the stowed configuration creates a short pressure profile, whereas the other two configurations create a longer pressure profile due to Fowler flap movement. The deflection leads to a suction peak on the flap, which also increases the suction on the entire wing up to the leading edge.
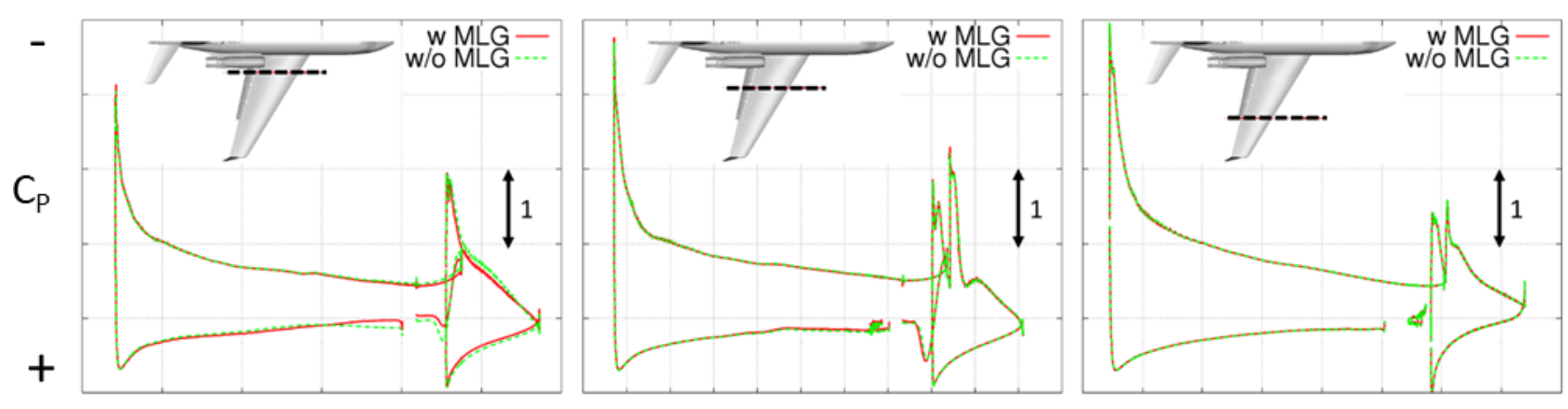

Figure 16 Pressure distribution at three different spanwise locations for simulation with (solid red) and without (dashed green) deployed MLG for flap deflection angle $\delta_{\mathrm{f}}=\mathbf{2 0}$. 

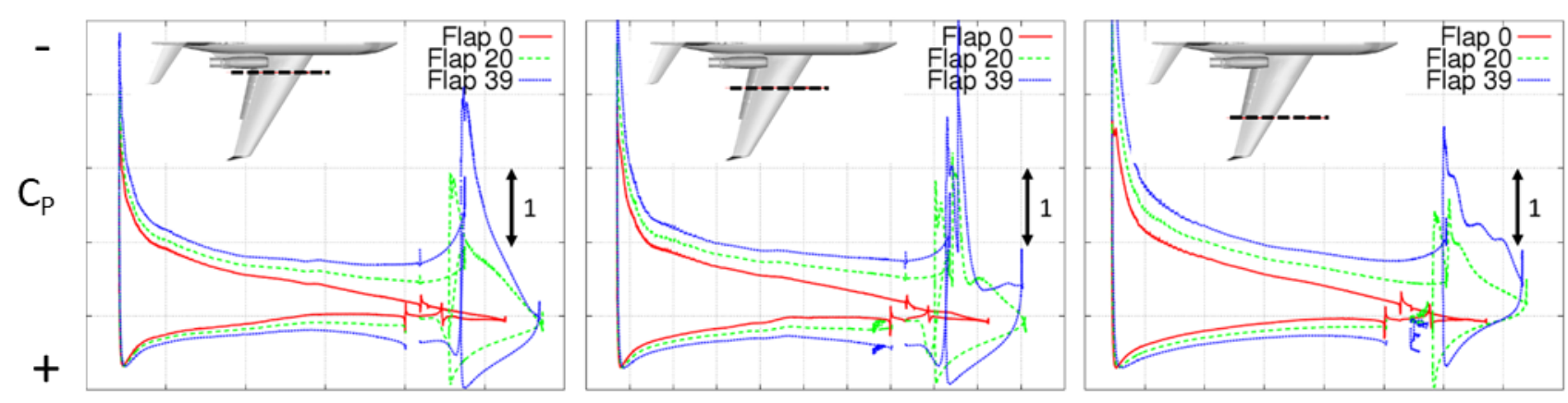

Figure 17 Pressure distribution at three different spanwise locations for simulation with stowed flap (solid red), flap deflected by $20^{\circ}$ (dashed green) and by $39^{\circ}$ (dotted blue).

For aircraft without leading edge slats (such as those manufactured by Gulfstream), airframe noise has been shown to be very weakly dependent on angle of attack within the linear lift regime [26]. As a result, even though the stowed configurations have been run at a slightly reduced angle of attack, the power spectral densities for a farfield pressure probe located below the aircraft at a distance of $120 \mathrm{~m}$ away give a good quantitative overview of the overall airframe noise characteristics, as shown in Figure 18. Comparing the impact of the MLG alone (solid red vs. dashed black line with +), a drastic increase of approximately $20 \mathrm{~dB}$ is seen across the entire spectrum. The shape of the curves, especially the decrease at high frequencies, is similar. Comparing the isolated impact of flap deflection (dashed green line vs. dash-dotted pink line vs. dashed black line with + ), a drastic increase over the entire frequency range becomes visible. This time, however, an additional effect at high frequencies can be observed. The drop-off now remains at a constant level due to the presence of strong noise sources at the flap side edges. However, we note that the sound levels and roll-off behavior at frequencies above $3.5 \mathrm{kHz}-4.0 \mathrm{kHz}$ may be questionable because of insufficient spatial resolution.

Looking now at the impact of gear deployment for two different flap settings (dashed green vs. double-dotted cyan and dashed pink vs. dotted blue), an increase is visible for both configurations at low to mid frequencies. The increase, however, is much more pronounced for the smaller flap deflection angle. High frequencies remain almost unchanged, highlighting the fact that flap side edge noise is dominant here. As discussed previously, the peak at about $550 \mathrm{~Hz}$ is attributed to a hollow post in the MLG. Interestingly, the peak diminishes with increasing flap deflection, probably as a result of diminished grazing flow velocity over the MLG cavity as a result of increased flap deflection due to the stagnation effect of the flap.

As expected, the highest broadband farfield noise is produced by the configuration with the largest flap deflection and a deployed landing gear. Nonetheless, this study highlights the complex interaction of main gear and flap side edge noise when these elements are in close proximity. Under these circumstances, the overall airframe noise must be predicted on a system level with high fidelity aeroacoustic methods, as was done here.

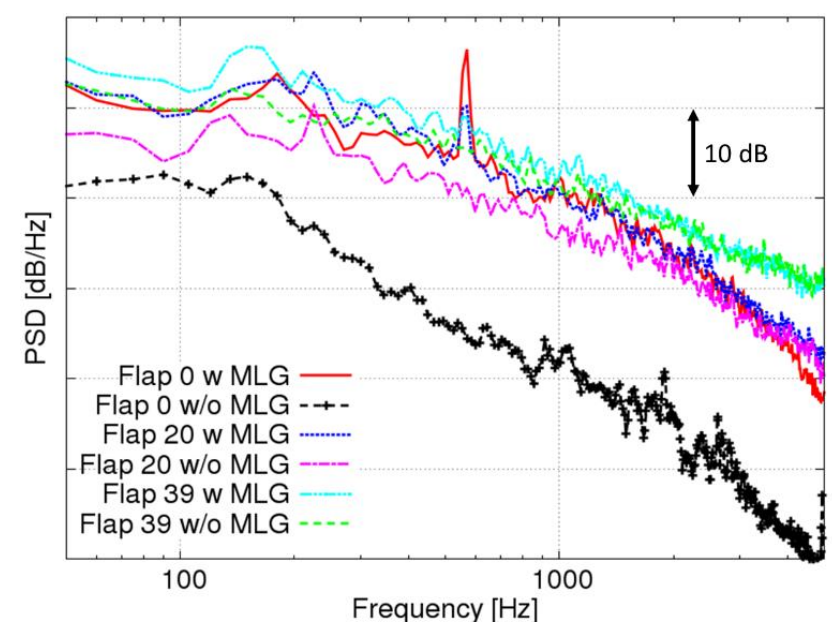

Figure 18 Power spectral density of farfield probe for various configurations. 


\section{Conclusion and Outlook}

A full simulation campaign for acquiring farfield noise data for the G-III aircraft in its baseline approach and landing configurations has been described. High geometrical fidelity was achieved by using laser-scanned definitions of the real aircraft surfaces and by including details, such as small hydraulic pipes, of the main landing gear and the flap bracket geometry. Results from simulations performed on coarse, medium and fine resolution grids were presented. Aerodynamic properties converged quickly whereas acoustic results, especially if high frequency data are needed, required higher mesh resolution. A study was performed to compare the solid and permeable FWH approach with a direct probe, which revealed a higher accuracy of the permeable FWH approach since volumetric effects can be captured. Full-span vs. half-span simulations were carried out revealing the existence of symmetrical and antisymmetrical main landing gear cavity modes. Finally, overall airframe noise characteristics were discussed, highlighting the necessity of high fidelity aeroacoustic simulations on a system rather than component level. This work will serve as basis for assessing landing gear noise reduction concepts in a future study.

\section{Acknowledgments}

This work was supported by the Flight Demonstrations and Capabilities (FDC) project under the Integrated Aviation Systems Program (IASP) of the NASA Aeronautics Research Mission Directorate. We would like to express our sincere appreciation to personnel at NASA Armstrong Flight Research Center for their assistance with development of the full-scale G-III aircraft geometry, particular Daniel Nolan and Michael Yandell of Jacobs Engineering for meticulously creating a high-definition CAD model for the main landing gear from the laser scan files. The authors also gratefully acknowledge the invaluable contribution of Scott Brynildsen of Craig Technologies for providing geometry modifications and CAD support. All the simulations were performed on the Pleiades supercomputer at the NASA Advanced Supercomputing (NAS) facility at Ames Research Center. The logistical support provided by NAS staff is greatly appreciated.

\section{References}

[1] Boeing, [Online]. Available: http://www.boeing.com/resources/boeingdotcom/commercial/about-ourmarket/assets/downloads/cmo_print_2016_final_updated.pdf. [Accessed 17 October 2017].

[2] M. Basner, C. Clark, A. Hansell, J. I. Hileman, S. Janssen, K. Shepherd and V. Sparrow, "Aviation Noise Impacts: State of the Science," Noise \& Health, vol. 19, no. 87, pp. 41-50, 2017.

[3] M. R. Khorrami, E. Fares and D. Casalino, "Towards Full Aircraft Airframe Noise Prediction: Lattice Boltzmann Simulations," AIAA Paper 2014-2481, 2014.

[4] E. Fares, D. Casalino and M. R. Khorrami, "Evaluation of Airframe Noise Reduction Concepts via Simulations Using a Lattice Boltzmann Approach," AIAA Paper 2015-2988, 2015.

[5] M. R. Khorrami and E. Fares, "Simulation-Based Airframe Noise Prediction of a Full-Scale Full Aircraft," AIAA Paper 2016-2706, 2016.

[6] E. J. Miller, J. Cruz, S. -F. Lung, S. Kota, G. Ervin, K. -J. Lu and P. Flick, "Evaluation of the Hinge Moment and Normal Force Aerodynamic Loads from a Seamless Adaptive Compliant Trailing Edge Flap in Flight," AIAA Paper 2016-0038, 2016.

[7] S. Kota, P. Flick and F. Collier, "Flight Testing of the FlexFloilTM Adaptive Compliant Trailing Edge," AIAA Paper 2016-0036, 2016.

[8] M. R. Khorrami, D. P. Lockard, J. W. M. Humphreys and P. A. Ravetta, "Flight-Test Evaluation of Airframe Noise Mitigation Technologies," in Paper to be presented at the AIAA/CEAS Aeroacoustics Conference in Atlanta, Georgia, June 2018, 2018.

[9] H. Chen, "Volumetric Formulation of the Lattice-Boltzmann Method for Fluid Dynamics: Basic Concept," Physical Review E, vol. 58, no. 3, pp. 3955-3963, 1998, doi: dx.doi.org/10.1103/PhysRevE.58.3955.

[10] H. Chen, C. Texeira and K. Molvig, "Realization of Fluid Boundary Condition via Discrete Boltzmann Dynamics," International Journal of Modern Physics C, pp. 1281-1292, 1998.

[11] H. Chen, S. Kandasamy, S. Orszag, R. Shock, S. Succi and V. Yakhot, "Extended Boltzmann Kinetic Equation for Turbulent Flows," Science, no. 301, pp. 633-636, 2003. 
[12] S. Chen and G. Doolen, "Lattice Boltzmann Method for Fluid Flows," Annual Review of Fluid Mechanics, vol. 30, pp. 329-364, 1998.

[13] E. Fares and S. Nölting, "Unsteady Flow Simulation of a High-Lift configuration using Lattice-Boltzmann Approach," AIAA Paper 2011-869, 2011.

[14] D. Casalino, S. Noelting, E. Fares, T. Van de Ven, F. Perot and G. Bres, "Towards Numerical Aircraft Noise Certification: Analysis of a Full-Scale Landing Gear in Fly-Over Configuration," AIAA Paper 2012-2235, 2012.

[15] D. Casalino, A. F. P. Ribeiro, E. Fares, S. Nölting, A. Mann, F. Perot, Y. Li, P.-T. Lew, C. Sun, P. Gopalakrishnan, R. Zhang, H. Chen and K. Habibi, "Towards Lattice-Boltzmann Prediction of Turbofan Engine Noise," AIAA Paper 2014-3101, 2014.

[16] H. Chen, S. Chen and W. H. Matthaeus, "Recovery of the Navier-Stokes equations using a lattice-gas Boltzmann method," Physical Review A, no. 45, pp. R5339-R5342, 1992.

[17] Y. H. Qian, D. D'Humières and P. Lallemand, "Lattice BGK Models for Navier-Stokes Equation," Europhysics Letters, vol. 17, pp. 479-484, 1992.

[18] V. Yakhot and S. Orszag, "Renormalization Group Analysis of Turbulence," Journal of Scientific Computing, vol. 1, no. 2, pp. 3-51, 1986, doi: 10.1007/BF01061452.

[19] E. Fares, "Unsteady Flow Simulation of the Ahmed Reference Body using a Lattice Boltzmann Approach," Journal of Computers and Fluids, no. 35, pp. 940-950, 2006,.

[20] E. Fares, B. Duda and M. R. Khorrami, "Airframe Noise Prediction of a Full Aircraft in Model and Full Scale Using a Lattice Boltzmann Approach," in AIAA Paper 2016-2707, 2016.

[21] J. E. Hawkings and D. L. Ffowcs Williams, "Sound Generation by Turbulence and Surfaces in Arbitrary Motion," Philosophical Transactions of the Royal Society of London. Series A, Mathematical and Physical Sciences, vol. 264, no. 1151, pp. 321-342, 1969.

[22] F. Farassat and G. Succi, "The Prediction of Helicopter Discrete Frequency Noise," Vertica, vol. 7, no. 4, pp. 309-320, 1983.

[23] A. Najafi-Yazdi, G. Brès and L. Mongeau, "An Acoustic Analogy Formulation for Moving Sources in Uniformly Moving Media," Proceeding of The Royal Society of London A, vol. 467, no. 2125, pp. 144-165, 2011.

[24] ICAO, Environmental Protection, Annex 16 to the Convention on International Civil Aviation, Vol. 1: Aircraft Noise, Appendix 2.

[25] B. König, E. Fares, P. Ravetta and M. Khorrami, "A Comparative Study of Simulated and Measured Main Landing Gear Noise for Large Civil Transports," AIAA Paper 2017-3013, 2017.

[26] M. R. Khorrami, J. W. M. Humphreys and D. P. Lockard, "An Assessment of Flap and Main Landing Gear Noise Abatement," in AIAA Paper 2015-2987, 2015. 